\title{
Biliary Atresia with Rare Collection of Congenital Anomalies
}

\author{
RMTM Gunawardena ${ }^{1}$, PAYP Weerawardhana ${ }^{2}$, AK Lamahewage ${ }^{3}$ \\ ${ }^{1}$ National Institute for Nephrology, Dialysis and Transplant, Maligawaththa, Sri Lanka; ${ }^{2}$ Base Hospital, Warakapola, Sri Lanka; \\ ${ }^{3}$ Lady Ridgeway Hospital for Children, Colombo 10, Sri Lanka.
}

\section{Corresponding Author:}

Dr. RMTM Gunawardena

Email: thilinamg@gmail.com

This is an Open Access article distributed under the terms of the Creative Commons Attribution License (creativecommons.org/ licenses/by/3.0).

Received : January 3, 2019

Accepted : May 24, 2019

Published : June 5, 2019

\begin{abstract}
Background: Biliary atresia (BA), characterized by progressive inflammatory destruction of the extra-hepatic biliary tree is one differential diagnosis for jaundice in the newborn. It is a diagnosis which should not be missed, as early surgical intervention is associated with better outcome. In upto $37 \%$ cases, this condition can be associated with other congenital anomalies. Case Report: A 2 month old baby girl diagnosed with biliary atresia was found to have a rare collection of coexisting anomalies such as dextrocardia, azygos continuation of the inferior vena cava, multiple spleens, central liver, right sided stomach and pre-duodenal portal vein. The baby underwent porto-enterostomy but she progressed to chronic liver failure. Conclusion: The associated anomalies with BA should be actively looked for in a child diagnosed with this condition, as they will affect the prognosis and the treatment approach.
\end{abstract}

Keywords: Biliary Atresia, End Stage Liver Disease, Enterostomy, Infant, Jaundice, Portal Vein.

\section{Introduction}

Biliary atresia (BA) is characterized by progressive inflammatory destruction of the extra-hepatic biliary tree with cholestasis [1]. It can present as an isolated entity but in upto $9-37 \%$ it is associated with other congenital anomalies. The polysplenia syndrome is the commonest such association [2]. Here we present a case report of a two month old baby girl with biliary atresia polysplenia syndrome with dextrocardia, pre-duodenal portal vein, right sided stomach and interrupted inferior vena cava (IVC).

\section{Case Report}

A two month old baby girl was referred to the pediatric surgical unit of Lady Ridgeway Hospital of Colombo, Sri Lanka for further management of cholestatic jaundice. Two weeks back, she was noted to have yellowish discoloration of her body with pale stools and ultrasound scan (USS) performed at the local hospital failed to demonstrate a gallbladder.

On examination at our hospital, she was jaundiced with stable vitals. There was hepatomegaly on abdominal palpation. Serum biochemistry revealed a conjugated hyper-bilirubinemia. The cardiac shadow was found to be on the right side of the chest on X-ray. Abdominal USS was repeated which confirmed the absence of the gallbladder. In addition, multiple spleens, a right sided stomach and a centrally placed liver were noted. Her 2D echocardiogram was compatible with dextrocardia and an additional finding of azygos continuation of the IVC was also detected.

A diagnosis of biliary atresia polysplenia syndrome was made and the child was scheduled for portoenterostomy. At laparotomy two right sided spleens [Fig.1], right sided stomach, central liver with macroscopic changes of cirrhosis were confirmed. The portal vein was found crossing 
in front of the duodenum [Fig.2]. The atretic gallbladder was dissected along with the portal plate and Rouex-en-Y portoenterostomy was performed. Following surgery, the child's bilirubin levels did not normalize and she progressed to chronic liver failure. At six months post-surgery she was under medical follow up for decompensated liver disease.

\section{Discussion}

Polysplenia is the presence of two or more spleens and when associated with other anomalies in chest and abdominal organs it is defined as the polysplenia syndrome (PS) [3]. Malrotation of the midgut, situs inversus, pre-duodenal portal vein, absent or interrupted infrahepatic IVC, abnormal hepatic arterial supply, central liver, cardiac and pulmonary anomalies have been described in those with PS [3]. In 1974 it was recognized that some patients with PS had biliary atresia and in 1993 the term biliary atresia splenic malformation syndrome was coined to describe this subset of patients [1]. The pathogenesis of biliary atresia polysplenia syndrome is thought to be due to an insult during organogenesis, occurring close to $5^{\text {th }}$ week of intrauterine life [1]. In those with isolated BA without other congenital abnormalities, the inciting cause probably acts after organogenesis is complete [4].

Surgical intervention for a child with BA polysplenia syndrome can be technically challenging due to associated intra-abdominal congenital anomalies and modification of the routine operative steps may become necessary $[1,4]$. Evaluation of complex anomalies with additional imaging modalities may take time and it is important to avoid undue delays in definitive surgical management as optimum results are achieved when a child with BA is operated within 8 weeks of age [4].

Studies comparing outcomes in operated children with isolated BA against those with the syndromic variant of the disease have shown that

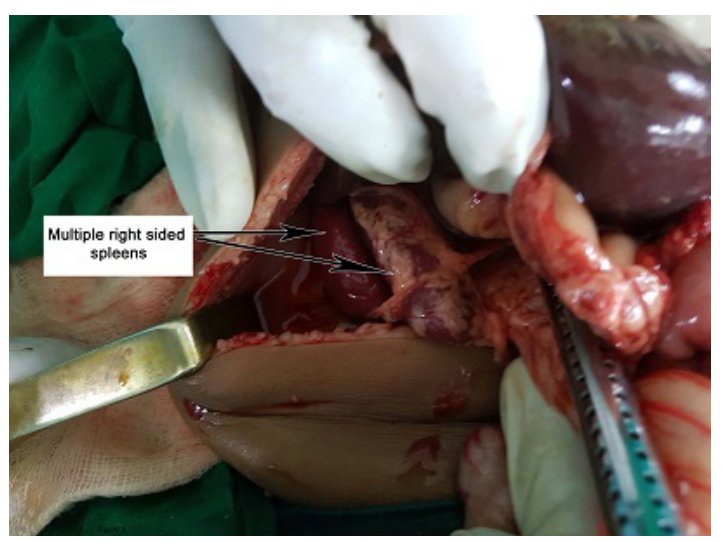

Fig.1: Multiple spleens seen on laparatormy.

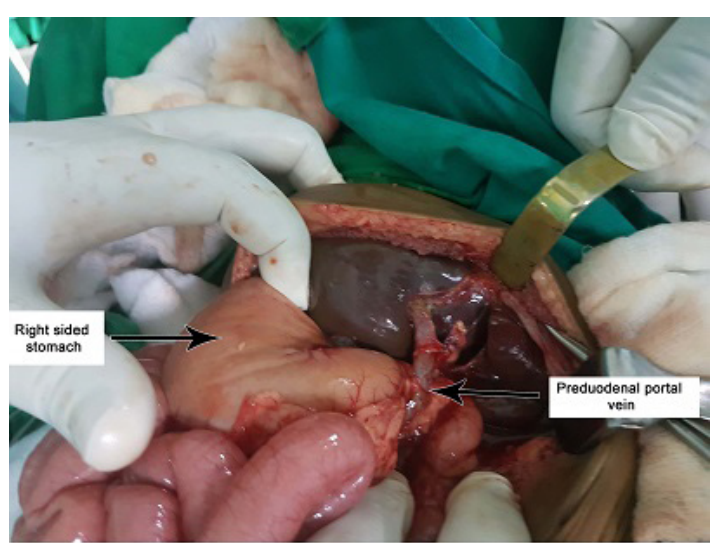

Fig.2: A right sided stomach, portal vein crossing in front of the duodenum.

the latter group fares worse, probably due to their associated anomalies [1]. However, there are studies which show comparable outcomes in both groups. Children with the syndromic variety of the disease are sick and they are detected and referred for specialized care relatively earlier compared to those with isolated BA, hence equal outcomes despite other anomalies [4].

\section{Conclusion}

Biliary atresia polysplenia syndrome is uncommon and affected individuals can have intra-abdominal anomalies which can make portoenterostomy challenging. Surgical intervention by experienced surgeons without undue delay will lead to better outcomes. 
Contributors: RMTMG: participated in the patient care, and writing the paper; PAYPW: patient care, manuscript writing. AKL participated in revising the manuscript critically and contributed towards writing discussion. RMTMG will act as guarantor. All authors approved the final version of this manuscript.

Funding: None; Competing interests: None stated.

\section{References}

1. Mathur P, Gupta R, Soni V, Ahmed R, Goyal RB. Biliary atresia associated with polysplenia syndrome, dextrocardia, situs inversus totalis and malrotation of intestines. Journal of Neonatal Surgery. 2014;3(1):9.
2. Varela-Fascinetto G, Castaldo P, Fox IJ, Sudan D, Heffron TG, Shaw BW, et al. Biliary atresia-polysplenia syndrome: surgical and clinical relevance in liver transplantation. Annals of Surgery. 1998;227(4):583589.

3. Rasool F, Mirza B. Polysplenia syndrome associated with situs inversus abdominus and type I jejunal atresia. APSP Journal of Case Reports. 2011;2(2):18.

4. Silveira TR, Salzana FM., Howard ER, Mowat AP. Congenital structural abnormalities in biliary atresia: evidence for etiopathogenic heterogeneity and therapeutic implications. Acta Paediatrica. 1991;80:1192-1199. 